\title{
Efecto de un dispositivo binivel autoajustable sobre los eventos respiratorios en pacientes con Síndrome de Apnea Obstructiva del Sueño
}

\author{
JOSÉ LUIS CARRILLO A.*,**, CÉSAR MANUEL SALAZAR P.*,**, \\ MARTHA GUADALUPE TORRES F.*,**, MARÍA DEL ROCÍO BAÑOS F.*,** y LUIS TORRE B.***
}

\section{Effect of an auto-bilevel device on respiratory events in patients with Obstructive Sleep Apnea Syndrome}

Objective: To evaluate the effect of an auto-bilevel device on respiratory events in adults with severe obstructive sleep apnea syndrome (OSAS). Polysomnographies (PSG) with the use of auto-bilevel device were reviewed. Sleep architecture, respiratory events, $\mathrm{O}_{2}$ saturation $\left(\mathrm{SpO} \mathrm{O}_{2}\right)$ and exhaled carbon dioxide (EtCO $\mathrm{O}_{2}$ ) were compared among baseline and therapeutic PSG. Results: We included 10 PSG. Auto-bilevel device corrected the sleep architecture; the apnea hypopnea index (AHI) decreased from 76 (39-137) to 14 (6-13) at the expense of obstructive and mixed apneas $(p<0.05)$, there was no significant decrease in hypopneas. Central apneas increased from $0.5(0-12.4)$ to $8.2(0-20) h^{-1}$. Sp $\mathrm{O}_{2}$ and EtCO $\mathrm{O}_{2}$ improved. Conclusions: In severe OSAS auto-bilevel device corrects sleep architecture, improves $\mathrm{SpO}_{2}$ and $\mathrm{EtCO}_{2}$ and decreases AHI at the expense of obstructive and mixed apneas, but could not eliminate hypopneas and even could increase central apneas.

Key words: Sleep apnea, obstructive; polysomnography; Adult; auto-bilevel device.

\section{Resumen}

Objetivo: Evaluar el efecto de un dispositivo binivel autoajustable sobre los eventos respiratorios en pacientes adultos con síndrome de apnea obstructiva del sueño (SAOS) grave. Se revisaron las polisomnografias (PSG) realizadas con el uso de un dispositivo de binivel autoajustable. La arquitectura de sueño, eventos respiratorios, saturación de $\mathrm{O}_{2}\left(\mathrm{SpO}_{2}\right)$ y dióxido de carbono exhalado (EtCO $\mathrm{C}_{2}$ ) se compararon entre la PSG basal y la PSG terapéutica. Resultados: Se incluyeron 10 PSG. El dispositivo binivel autoajustable corrigió la arquitectura de sueño; disminuyó el índice de apnea hipopnea (IAH) de 76 (39-137) a 14 (6-13) a expensas de apneas obstructivas y mixtas $(p<0,05)$, no se observó descenso significativo en las hipopneas. Las apneas centrales incrementaron de 0,5 $(0-12,4)$ a 8,2 (0-20) $h^{-1}$. La SpO y EtCO mejoraron. Conclusiones: En pacientes con SAOS grave el dispositivo binivel autoajustable corrige la arquitectura de sueño, mejora la $\mathrm{SpO}_{2}$ y EtCO $\mathrm{CO}_{2}$ disminuye el IAH a expensas de apneas obstructivas y mixtas, pero podría no eliminar las hipopneas e incrementar las apneas centrales.

Palabras clave: Apnea obstructiva del sueño, polisomnografía, adulto, dispositivo binivel autoajustable.

\footnotetext{
* Unidad de Medicina de Sueño, Instituto Nacional de Enfermedades Respiratorias Ismael Cosio Villegas, Ciudad de México, México.

** Academia Mexicana de Medicina del Dormir AC.
} 


\section{Introducción}

El Síndrome de Apnea Obstructiva del Sueño (SAOS) es un trastorno caracterizado por un número anormal de episodios intermitentes en los que se interrumpe en forma total o parcial el flujo respiratorio durante el dormir ${ }^{1}$; es secundario al colapso/cierre de la vía área superior (VAS), está asociado a complicaciones cardiovasculares y representa un problema global de salud pública ${ }^{2}$.

El estándar de tratamiento es dormir con un dispositivo de presión positiva continua en la vía aérea (CPAP por sus siglas en inglés), el cual administra presión positiva que mantiene abierta la VAS evitando así su colapso, esta presión es frecuentemente titulada durante una polisomnografía (PSG). Sin embargo, un dispositivo de CPAP automático o auto-ajustable puede obviar este procedimiento ${ }^{3,4}$. Existen dispositivos de dos presiones o binivel que proporcionan una presión mayor durante la inspiración (IPAP, siglas en inglés) y una presión menor durante la espiración (EPAP, siglas en inglés) que también se puede usar como tratamiento del SAOS, su indicación más frecuente es una presión terapéutica $\geq 15 \mathrm{~cm} \mathrm{H}_{2} \mathrm{O}$ de $\mathrm{CPAP}^{5}$; desde hace varios años están disponibles comercialmente los dispositivos binivel automáticos o autoajustables, estos equipos cuentan con un algoritmo que detecta los eventos respiratorios y modifica automáticamente sus dos presiones hasta eliminarlos, pero a pesar de utilizarse con cierta frecuencia su desempeño como tratamiento del SAOS ha sido poco evaluado, así, el objetivo de este estudio fue evaluar el efecto de un dispositivo binivel autoajustable sobre los eventos respiratorios en pacientes con SAOS grave.

\section{Materiales y Métodos}

Este estudio fue aprobado por los comités de ciencia y bioética en investigación institucionales (Código C04-17).

Se revisaron las polisomnografías realizadas en el Laboratorio de la Unidad de Medicina de Sueño del Instituto Nacional de Enfermedades Respiratorias (INER) Ismael Cosío Villegas, desde el $1^{\circ}$ de enero de 2006 hasta el 31 de diciembre de 2016; se incluyeron los registros de pacientes que cumplieron los siguientes criterios: edad $\geq 18$ años, índice de apnea hipopnea $\geq 30$ eventos por hora de sueño, más del $50 \%$ de los eventos de tipo obstructivo y que durante la titulación se les hubiera colocado un dispositivo binivel autoajustable. Se excluyeron a los re- gistros con eficiencia de sueño $<55 \%$ así como pacientes con narcolepsia, movimiento periódico de extremidades y cualquier otro diagnóstico diferente a SAOS.

Se revisaron 5.103 polisomnografías de las cuales diez cumplieron con los criterios de selección. En todos los pacientes se contó con polisomnografía basal y polisomnografía ventilatoria lo que permitió hacer una comparación pre versus post binivel auto-ajustable. El montaje utilizado en todas las polisomnografías incluidas fue el siguiente: electroencefalograma (F4M1, C4M1, $\mathrm{O} 2 \mathrm{M} 1$ ), electro-oculografia bilateral, electromiografía de mentón, flujo respiratorio (cánula de presión nasal y sensor térmico oronasal en el caso del estudio basal y flujo de binivel en el caso del registro ventilatorio), dióxido de carbono exhalado $\left(\mathrm{EtCO}_{2}\right)$, pulsoximetría $\left(\mathrm{SpO}_{2}\right)$, banda en tórax y abdomen (pletismografía por inductancia), electrocardiograma (DII) y electromiografía de tibial anterior. Los estudios fueron calificados manualmente por personal experimentado de acuerdo a las reglas de la Academia Americana de Medicina del Sueño (AAMS) vigentes para el momento ${ }^{6}$.

En todas las PSG ventilatorias incluidas y analizadas se colocó un equipo BiPAP Auto serie $\mathrm{M}^{\circledR}$ (Respironics $^{\circledR}$ ), este equipo cuenta con un algoritmo basado en vibración y flujo para identificar a las apneas e hipopneas y entrega terapia binivel en modo espontáneo con un ajuste automático de IPAP y EPAP de acuerdo a las necesidades del paciente, brevemente el algoritmo automático de este equipo funciona de la siguiente manera: a) si se detectan 2 apneas obstructivas consecutivas en un período de 3 min se incrementa la EPAP en $1 \mathrm{~cm} \mathrm{H}_{2} \mathrm{O}$; b) en caso de presentar hipopneas o limitación al flujo el algoritmo aumenta la IPAP en $1 \mathrm{~cm} \mathrm{H}_{2} \mathrm{O}$ hasta eliminar el evento o hasta que el nivel máximo de presión sea alcanzado; c) cada incremento de IPAP genera un aumento equivalente de EPAP para mantener una presión soporte constante ${ }^{7}$. En todos los casos incluidos el equipo fue programado con presiones abiertas de la siguiente manera: EPAP mínimo $4 \mathrm{~cm} \mathrm{H}_{2} \mathrm{O}$, IPAP máximo $25 \mathrm{~cm} \mathrm{H}_{2} \mathrm{O}$, presión soporte mínima $3 \mathrm{~cm} \mathrm{H}_{2} \mathrm{O}$ (este parámetro ya viene definido en el equipo y no puede ser modificado), presión soporte máxima $8 \mathrm{~cm} \mathrm{H}_{2} \mathrm{O}$ con una mascarilla nasal adaptada para cada paciente, en ningún caso se utilizó oxígeno suplementario.

De las polisomnografías se registró la siguiente información: arquitectura de sueño, eventos respiratorios, saturación de oxígeno y dióxido de carbono exhalado.

Debido a su distribución las variables se re- 
sumieron como medianas (mínimo-máximo). La comparación de datos relacionados se realizó con prueba de rangos señalados de Wilcoxon mientras que los independientes con la prueba U-Mann Whitney. La significancia estadística se estableció con valor de $\mathrm{p}<0,05$ bimarginal. Se utilizó el paquete estadístico STATA 12.

\section{Resultados}

Todos los pacientes fueron hombres, obesos, tenían somnolencia excesiva diurna, $\mathrm{SpO}_{2}$ diurna $>90 \%$ y no habían recibido nunca un dispositivo de presión positiva, las características generales de los pacientes se presentan en la Tabla 1.

El dispositivo binivel autoajustable a pesar de aumentar la latencia a sueño, ocasionó cambios significativos en la arquitectura de sueño como disminución de N2, aumento de N3, aumento de $\mathrm{R}$ y la disminución del índice de alertamientos (este último parámetro a expensas de alertamientos respiratorios). La comparación completa de la arquitectura de sueño entre la PSG basal y la PSG con el uso de dispositivo binivel autoajustable se presenta en la Tabla 2.

En la PSG con dispositivo binivel autoajustable se observó un descenso significativo del índice de apnea hipopnea (IAH), parámetro que define la gravedad en el paciente con SAOS, de 76 (39-137) a 14 (6-13) eventos por hora de sue- ño $\left(\mathrm{h}^{-1}\right)$. Sin embargo, solo 3 pacientes obtuvieron una titulación adecuada (IAH $<10 \mathrm{~h}^{-1}$ ) y ninguno una titulación óptima (IAH $<5 \mathrm{~h}^{-1}$ ), vide infra ${ }^{5}$. La disminución del IAH fue a expensas de apneas obstructivas: 41,6 (4-115) versus 2,6 (0-6) $\mathrm{h}^{-1} \mathrm{y}$

Tabla 1. Características generales de los pacientes con SAOS grave

\begin{tabular}{|c|c|}
\hline & $\begin{array}{c}\text { Mediana } \\
\text { (mínimo-máximo) }\end{array}$ \\
\hline Edad (años) & $38(27-61)$ \\
\hline Peso (kg) & $89,8(69-122)$ \\
\hline Talla (m) & $1,6(1,6-1,8)$ \\
\hline $\operatorname{IMC}\left(\mathrm{kg} / \mathrm{m}^{2}\right)$ & $33(26,3-39)$ \\
\hline Circunferencia cervical (cm) & $44(40-47)$ \\
\hline Circunferencia abdominal (cm) & $107(80-126)$ \\
\hline Presión arterial sistólica (mmHg) & $120(90-180)$ \\
\hline Presión arterial diastólica $(\mathrm{mmHg})$ & $80(60-110)$ \\
\hline Frecuencia cardiaca (lpm) & $75(55-86)$ \\
\hline $\mathrm{SpO}_{2}(\%)$ & $93(92-95)$ \\
\hline $\mathrm{EtCO}_{2}$ vigilia $(\mathrm{mmHg})$ & $38(33-45)$ \\
\hline Escala de Epworth & $19(12-24)$ \\
\hline SACS & $50(40-57)$ \\
\hline
\end{tabular}

Abreviaturas: IMC: índice de masa corporal, lpm: latidos por minuto, SACS: Sleep apnea clinical score, $\mathrm{SpO}_{2}=\mathrm{sa}-$ turación arterial de oxígeno, $\mathrm{EtCO}_{2}: \mathrm{CO}_{2}$ espirado.

Tabla 2. Comparación de la arquitectura de sueño entre la polisomnografía basal y la ventilatoria con dispositivo binivel autoajustado

\begin{tabular}{|lccc|}
\hline & $\begin{array}{c}\text { PSG Basal } \\
\text { Mediana } \\
\text { (mínimo-máximo) }\end{array}$ & $\begin{array}{c}\text { PSG Binivel autoajustado } \\
\text { Mediana } \\
\text { (mínimo-máximo) }\end{array}$ & p \\
\hline TST (min) & $446(442-525)$ & $394(327-491)$ & 0,68 \\
\hline Latencia a sueño (min) & $20(1-42)$ & $36,7(12-63)$ & $\mathbf{0 , 0 0 5}$ \\
\hline Latencia a R (min) & $149(75-349)$ & $90(3-292)$ & 0,09 \\
Eficiencia (\%) & $83(58-98)$ & $76(43-95)$ & 0,74 \\
\hline R (\% TST) & $11(5,8-19,2)$ & $24,3(14-46)$ & $\mathbf{0 , 0 1}$ \\
N1 (\% TST) & $20,2(3,1-36)$ & $11,6(3-30)$ & 0,06 \\
\hline N2 (\% TST) & $55,3(49,9-77,5)$ & $38(28-60)$ & $\mathbf{0 , 0 2}$ \\
N3 (\% TST) & $6,1(0-23,7)$ & $22(3-33)$ & $\mathbf{0 , 0 2}$ \\
IA ( $\left.\mathrm{h}^{-1}\right)$ & $63(18-117)$ & $17(3-25)$ & 0,007 \\
IAR $\left(\mathrm{h}^{-1}\right)$ & $54(4,6-108)$ & $13(1-16,7)$ & $\mathbf{0 , 0 0 5}$ \\
\hline IAE $\left(\mathrm{h}^{-1}\right)$ & $4,75(0-11,7)$ & $6(0,4-15)$ & 0,16 \\
\hline
\end{tabular}

Abreviaturas: $\mathrm{h}^{-1}=$ eventos por hora de sueño, $\mathrm{IA}=$ índice de alertamientos, $\mathrm{IAE}=$ índice de alertamientos espontáneos, $\mathrm{IAR}=$ índice de alertamientos respiratorios, $\min =$ minutos, $\mathrm{N} 1=$ estadio 1 de sueño, N2 = estadio 2 de sueño, N3 = estadio 3 de sueño, $\mathrm{PSG}=$ polisomnografía, $\mathrm{R}=$ sueño de movimientos oculares rápidos, $\mathrm{TST}=$ tiempo total de sueño. 
Tabla 3. Comparación de los eventos respiratorios, intercambio gaseoso y frecuencia cardiaca entre la PSG basal y la terapéutica

\begin{tabular}{|lccl|}
\hline & $\begin{array}{c}\text { PSG Basal } \\
\text { Mediana (mínimo-máximo) }\end{array}$ & $\begin{array}{c}\text { PSG Binivel autoajustado } \\
\text { Mediana (mínimo-máximo) }\end{array}$ & p \\
\hline $\mathrm{IAH}\left(\mathrm{h}^{-1}\right)$ & $76(39-137)$ & $14(6-33)$ & $\mathbf{0 , 0 0 5}$ \\
$\mathrm{IAC}\left(\mathrm{h}^{-1}\right)$ & $0,5(0-12,4)$ & $8,2(0-20)$ & $\mathbf{0 , 0 2}$ \\
\hline $\mathrm{IAO}\left(\mathrm{h}^{-1}\right)$ & $41,6(4-115)$ & $2,65(0-6)$ & $\mathbf{0 , 0 0 5}$ \\
$\mathrm{IAM}\left(\mathrm{h}^{-1}\right)$ & $18,5(0-37)$ & $0,3(0-6,6)$ & 0,17 \\
$\mathrm{IHIP}\left(\mathrm{h}^{-1}\right)$ & $6,4(0-26,1)$ & $5(1-12)$ & 0,38 \\
$\mathrm{~T} 90 \%(\%)$ & $68(13-99)$ & $10,7(1-74)$ & $\mathbf{0 , 0 0 8}$ \\
$\mathrm{SpO}_{2}(\%)$ & $82,5(65-92)$ & $91(87-95)$ & $\mathbf{0 , 0 0 8}$ \\
$\mathrm{SpO}_{2} \mathrm{R}(\%)$ & $76(56-92)$ & $91(88-93)$ & $\mathbf{0 , 0 0 7}$ \\
$\mathrm{SpO}_{2} \mathrm{~N}$ & $82,5(67-92)$ & $91(86-94)$ & $\mathbf{0 , 0 0 8}$ \\
$\mathrm{ID}\left(\mathrm{h}^{-1}\right)$ & $77,8(13-99)$ & $15(5-41,5)$ & $\mathbf{0 , 0 1}$ \\
$\mathrm{ID} \mathrm{R}\left(\mathrm{h}^{-1}\right)$ & $47(7-83)$ & $5(1,7-48,7)$ & $\mathbf{0 , 0 0 9}$ \\
$\mathrm{ID} \mathrm{N}\left(\mathrm{h}^{-1}\right)$ & $81(15-102)$ & $16.5(6-49,5)$ & 0,17 \\
\hline $\mathrm{EtCO}$ & máximo & $46(41-52)$ & $\mathbf{0 , 0 1}$ \\
$\mathrm{FC} \mathrm{R}(\mathrm{lpm})$ & $51,5(52-64)$ & $61(50-68)$ & 0,06 \\
\hline $\mathrm{FC} \mathrm{N}(\mathrm{lpm})$ & $73(53-101)$ & $63,6(52-68)$ & 0,07 \\
\hline
\end{tabular}

Abreviaturas: $\mathrm{EtCO}_{2}=$ dióxido de carbono exhalado, $\mathrm{h}^{-1}=$ eventos por hora de sueño, IAC = índice de apnea central, IAH, índice de apnea hipopnea, IAM = índice de apnea mixta, IAO = índice de apnea obstructiva, IHIP = índice de hipopneas, $\mathrm{lpm}=$ latidos por minuto, $\mathrm{mmHg}=$ milímetros de mercurio, $\mathrm{N}=$ sueño de no movimientos oculares rápidos, $\mathrm{R}=$ sueño de movimientos oculares rápidos, $\mathrm{SpO}_{2}=$ saturación, $\mathrm{T} 90$ = tiempo de saturación por debajo de $90 \%$. $\mathrm{FC}=$ frecuencia cardiaca.

apneas mixtas: $18,5(0-37)$ versus $0,3(0-6,6) \mathrm{h}^{-1}$ con $\mathrm{p}<0,05$; sin embargo, en las hipopneas no se demostró una disminución significativa $6,4(0-$ $26,1)$ versus $5(1-12) \mathrm{h}^{-1} \mathrm{p}=0,38 \mathrm{y}$ en las apneas centrales se presentó incluso un incremento significativo de $0,5(0-12,4)$ a $8,2(0-20) \mathrm{h}^{-1}(\mathrm{p}=0,02)$.

Con respecto al intercambio gaseoso, el dispositivo binivel autoajustable mejoró en forma significativa la oxigenación nocturna expresada por el $\mathrm{T} 90 \%$ (tiempo de $\mathrm{SpO}_{2}<90 \%$ expresado en porcentaje del tiempo total de sueño) y $\mathrm{SpO}_{2}$ promedio durante el sueño, cabe destacar que esta mejoría tiende a ser mayor en el sueño de movimientos oculares rápidos (mediana de diferencia de $\mathrm{SpO}_{2}$ promedio de $15,5 \%, 1-33$ ) en comparación con el sueño de no movimientos oculares rápidos (mediana de diferencia de $\mathrm{SpO}_{2}$ promedio de $7 \%, 0-18) p=0,17$. Definiendo oxemia normal durante el sueño como al constructo de T90\% $<30 \%$ y $\mathrm{SpO}_{2}$ promedio $\geq 90 \%$ en la PSG ventilatoria, el dispositivo binivel autoajustable normalizó este parámetro en 8 de los 10 pacientes.

Acerca de la ventilación durante el sueño, el dispositivo binivel autoajustable aminoró el $\mathrm{EtCO}_{2}$ máximo (dióxido de carbono exhalado máximo alcanzado durante la noche) de 51,5 (52-
64) a 46 (41-52) mmHg; en 5 de los 10 pacientes el $\mathrm{EtCO}_{2}$ máximo se disminuyó a un nivel $<45$ $\mathrm{mmHg}$. La comparación completa de los eventos respiratorios y el intercambio gaseoso durante el sueño se presentan en la Tabla 3.

No se encontraron diferencias significativas en la frecuencia cardiaca tanto en sueño R como en sueño N entre la PSG basal y la PSG ventilatoria.

\section{Discusión}

De la información desprendida de este artículo destaca que el dispositivo binivel autoajustable es un modo de presión positiva muy poco utilizado en el Laboratorio de la Unidad de Medicina de Sueño del Instituto Nacional de Enfermedades Respiratorias. Así, en 10 años y más de 5 mil estudios solo se localizaron 10 polisomnografìas que cumplieran los criterios de selección. Este estudio demuestra además que el equipo binivel auto-ajustable corrige la macro y la microarquitectura de sueño, modifica los eventos respiratorios y mejora el intercambio gaseoso durante el sueño, todo esto en sujetos con apnea obstructiva del sueño grave. 
Acerca de los cambios en la macro y microarquitectura de sueño se observaron cambios que definen mejoría en la calidad de sueño, como la disminución de sueño superficial (N2), aumento de sueño profundo (N3), incremento de sueño de movimientos oculares rápidos $(\mathrm{R})$ y un descenso considerable del índice de alertamientos respiratorios; todo atribuible a los cambios ejercidos por el equipo sobre los eventos respiratorios (vide infra). Sin embargo, cabe destacar que el binivel prolongó en algunos minutos la latencia a sueño y no modificó la latencia a sueño $\mathrm{R}$ ni la eficiencia de sueño, posiblemente como reflejo de la incomodidad de usar una mascarilla; esta mejoría ha sido ampliamente reportada en el caso de CPAP $^{8}$. Sin embargo, con el equipo binivel autoajustable los reportes son limitados; en un estudio de asignación aleatoria, llevado a cabo en Berlín, Alemania (altura media de 32 metros sobre el nivel medio del mar, msnmm), 17 adultos con apnea obstructiva del sueño grave recibieron tratamiento con un equipo binivel autoajustable (BiPAP ${ }^{\circledR}$ Auto con Bi-Flex Philips Respironics) a presiones abiertas con liberación de presión, se describen los cambios producidos en la arquitectura de sueño, sin embargo, no hacen una comparación estadística, en este mismo estudio el IAH se redujo de 39,3 $\pm 15,2$ a 4,8 $\pm 3,6$ eventos por hora de sueño 9 .

En relación a los eventos respiratorios, el equipo binivel autoajustable redujo significativamente el índice de apnea hipopnea, este hallazgo ya había sido reportado con anterioridad, así, en un estudio observacional ejecutado en Lille, Francia (a una altitud de $21 \mathrm{msnmm}$ ) que evaluó a 35 pacientes con SAOS sin apego a tratamiento previo con CPAP recibieron un equipo binivel autoajustable (BiPAP ${ }^{\circledR}$ Auto con Bi-Flex Philips Respironics) como una estrategia para incrementar las horas de uso, reportaron una disminución significativa del IAH de 39,6 (25-58,6, min-max) a $3,3(1,1-9)$ por hora de sueño ${ }^{10}$, y en un estudio que incluyó pacientes habitantes de Pavia, Italia (altitud $77 \mathrm{msnmm}$ ) con apnea del sueño grave, 35 no respondedores a CPAP y 31 que no toleraron el mismo, se observó una disminución del IAH a 4,6 $\pm 4,9$ y $4,2 \pm 3,3 \mathrm{~h}^{-1}$ respectivamente $^{7}$ después de recibir un Binivel auto Respironics.

Cabe mencionar que en todas las series anteriores el equipo binivel autoajustable logró disminuir el IAH a $<5 \mathrm{~h}^{-1}$, nivel de tratamiento descrito como óptimo de acuerdo a los criterios de la $\mathrm{AAMS}^{5}$. Sin embargo, su desempeño en nuestros pacientes varió de acuerdo al tipo de eventos respiratorios. Así, hubo una eliminación casi absoluta de las apneas obstructivas y mixtas; sin embargo, no se demostró el mismo efecto sobre las hipopneas ni en las apneas centrales, en este último caso incluso hubo un incremento. Los equipos autoajustables pueden responder en forma diferente dependiendo de su algoritmo y tecnología ${ }^{11}$, en todos los casos descritos se utilizó un equipo que se basa en vibración y limitación al flujo, pero carece de oscilometría, de tal manera, pareciera que el dispositivo detectó y respondió adecuadamente a obstrucciones totales como las apneas obstructivas y mixtas; sin embargo, en obstrucciones parciales (hipopneas) podría no haber respondido adecuadamente y no modificó así su presión para eliminar los eventos; en relación al incremento en el índice de apneas centrales la ausencia de oscilometría pudo haber provocado que el equipo no diferenciara entre apneas obstructivas y centrales y así este pudo incrementar la presión de soporte (diferencia entre la presión inspiratoria y la espiratoria) innecesariamente favoreciendo la aparición de apneas centrales emergentes a tratamiento ${ }^{1}$, además, este mecanismo pudo estar exacerbado por la altitud de la Ciudad México que se encuentra a $2.240 \mathrm{msnmm}$, dado que los estudios anteriores se ejecutaron en centros que se encuentran prácticamente a nivel del mar y la altura moderada es un factor de riesgo para desarrollar este tipo de eventos respiratorios durante el sueño ${ }^{12}$; cabe destacar, que esta manera de responder ante los eventos puede tener impacto en la efectividad del tratamiento. Así, solo tres de los diez pacientes obtuvieron una titulación adecuada y ninguno una titulación óptima poniendo en duda la pertinencia de su indicación.

Con respecto al intercambio gaseoso también se observó una mejoría significativa, en el estado de oxigenación se observaron los cambios más importantes y 8 de los 10 pacientes consiguieron una normalización de este parámetro; sin embargo, solo en 5 paciente se logró disminuir el $\mathrm{EtCO}_{2}$ hasta un umbral menor a $45 \mathrm{mmHg}$ (umbral ajustado para hipoventilación durante el sueño en residentes a altitudes moderadas) ${ }^{13}$; la causa de estos hallazgos nuevamente podría ser consecuencia del equipo, el cual está diseñado para eliminar evento obstructivos, pero no para mantener un volumen corriente objetivo y estabilizar así la ventilación. Sin embargo, los autores de este trabajo consideran que este equipo debió normalizar la ventilación nocturna en este grupo de pacientes.

Este estudio tiene varias limitaciones, como el carácter restrospectivo del diseño y el número reducido de casos incluidos producto de la política del Laboratorio de la Unidad de Medicina de 
Sueño del INER de no utilizar equipos autoajustables durante las polisomnografías terapéuticas. Sin embargo, las otras series no son mucho mayores; se utilizó un equipo binivel autoajustable con variables de fase automáticas y no se evaluaron posibles asincronías paciente/ventilador y su posible efecto sobre los eventos respiratorios, la oxemia y el $\mathrm{EtCO}_{2}$. Sin embargo, en un estudio donde se compararon siete diferentes equipos binivel evaluados con un simulador robotizado, el equipo utilizado tuvo un buen desempeño en mantener un volumen corriente estable y tiempo libre de asincronias ${ }^{11}$. La principal ventaja de esta investigación fue el evaluar polisomnografías pre y post equipo binivel autoajustable lo que permitió comparar el efecto del dispositivo sobre los eventos respiratorios, la arquitectura de sueño y el intercambio gaseoso usando el estándar de referencia, a diferencia del resto de los estudios que usaron la tarjeta de memoria de los equipos, además, en todos los casos se utilizó el mismo equipo con programación idéntica incrementando la validez externa de los resultados.

\section{Conclusiones}

En adultos con Síndrome de Apnea Obstructiva del Sueño grave el equipo binivel autoajustable evaluado corrigió la arquitectura de sueño, mejoró la $\mathrm{SpO}_{2}$ y el $\mathrm{EtCO}_{2}$ y disminuyó el Índice de Apnea Hipopnea, este último a expensas de apneas obstructivas y mixtas, pero podría no eliminar las hipopneas e incrementar el índice de apneas centrales.

\section{Bibliografía}

1.- AMERICAN ACADEMY OF SLEEP MEDICINE. International Classification of Sleep Disorders, third edition. Darien Il. American Academy of Sleep Medicine, 2014.

2.- CARRILLO-ALDUENDA JL, ARREDONDO-DELBOSQUE FM, REYEZ-ZÚÑIGA MM, CASTORENAMALDONADO AR, VÁZQUEZ-GARCÍA JC, TORRE-BOUSCOULET L. Síndrome de apnea obstructiva del sueño en población adulta. Neumol Cir Torax 2010; 69: 103-15.

3.- EPSTEIN LJ, KRISTO D, STROLLO PJ JR, FRIEDMAN N, MALHOTRA A, PATIL SP, et al. Clinical guideline for the evaluation, management and long-term care of obstructive sleep apnea in adults. J Clin Sleep Med 2009; 5: 263-76.

4.- KUSHIDA CA, LITTNER MR, MORGENTHALER T, ALESSI CA, BAILEY D, COLEMAN J Jr, et al. Practice parameters for the indications for polysomnography and related procedures: an update for 2005. Sleep 2005; 28: 499-521.

5.- KUSHIDA CA, CHEDIAK A, BERRY RB, BROWN L $\mathrm{K}$, GOZAL D, IBER C, et al. Clinical guidelines for the manual titration of positive airway pressure in patients with obstructive sleep apnea. J Clin Sleep Med 2008; 15: $157-71$.

6.- IBER C, ANCOLI-ISRAEL S, CHESSON AL, QUAN $\mathrm{S}$. The AASM manual for the scoring of sleep and associated events: rules, terminology, and technical specifications, $1^{\text {st }}$ ed. Westchester, IL: American Academy of Sleep Medicine, 2007.

7.- CARLUCCI A, CERIANA P, MANCINI M, CIRIO S, PIERUCCI P, D'ARTAVILLA LUPO N, et al. Efficacy of bilevel-auto treatment in patients with obstructive sleep apnea not responsive to or intolerant of continuos positive airway pressure ventilation. J Clin Sleep Med 2015; 11: 981-5.

8.- MCARDLE N, DOUGLAS NJ. Effect of continuous airway pressure on sleep architecture in the sleep apneahypopnea syndrome: a randomized controlled trial. Am J Respir Crit Care Med 2001; 164: 1459-63.

9.- BLAU A, MINX M, PETER JG, GLOS M, PENZEL T, BAUMANN G, et al. Auto bilevel pressure relief-PAP is as effective as CPAP in OSA patients-a pilot study. Sleep Breath 2012; 16: 773-9.

10.- GENTINA T, FORTIN F, DOUAY B, DERNIS JM, HERENGT F, BOUT JC, et al. Auto bi-level with pressure relief during exhalation as a rescue therapy for optimally treated obstructive sleep apnoea patients with poor compliance to continuous positive airway pressure therapy-a pilot study. Sleep Breath 2011; 15: 21-7.

11.- CHEN Y, CHENG K, ZHOU X. Performance of seven bilevel mechanical ventilators in pressure-support mode with different cycling criteria: A comparative bench study. Med Sci Monit 2015; 21: 310-7.

12.- NUSSBAUMER-OCHSNER Y, SCHUEPFER N, ULRICH S, BLOCH K E. Exacerbation of sleep apnoea by frequent central events in patients with the obstructive sleep apnoea syndrome at altitude: a randomised trial. Thorax 2010; 65: 429-35.

13.- MATHEUS-RAMÍREZ EP, BELLO-CARRERA R S, TORRES-FRAGA MG, ARIAS-ARIAS PA, RAMÍREZ-ANAYA SN, BAZURTO-ZAPATA MA, et al. Comentarios Clínicos a la $3^{\text {ra }}$ Clasificación Internacional de los Trastornos Respiratorios del Dormir. Tercera Parte: Trastornos de hipoventilación relacionados al sueño. Respirar 2017; 9: 20-6.

Correspondencia a:

Dr. José Luis Carrillo Alduenda

Unidad de Medicina de Sueño, Instituto Nacional de Enfermedades Respiratorias Ismael Cosio Villegas, Calzada Tlalpan 4502, Colonia sección XVI,

Delegación Tlalpan, Ciudad de México, México, CP 14080.

Email: jlcarrillo14@hotmail.com 